\title{
A decomposable silica-based antibacterial coating for percutaneous titanium implant
}

\author{
This article was published in the following Dove Press journal: \\ International Journal of Nanomedicine \\ 6 January 2017 \\ Number of times this article has been viewed
}

\begin{abstract}
Jia Wang, ${ }^{1, *}$ Guofeng Wu, ${ }^{2, *}$ Xiangwei Liu, ${ }^{3, *}$ Guanyang Sun,' Dehua Li, ${ }^{3}$ Hongbo Wei ${ }^{3}$

'State Key Laboratory of Military Stomatology, National Clinical Research Center for Oral Diseases \& Shaanxi Key Laboratory of Stomatology, Department of Prosthodontics, School of Stomatology, The Fourth Military Medical University, Xi'an, ${ }^{2}$ Department of Prosthodontics, Nanjing Stomatological Hospital, Medical School of Nanjing University, Nanjing, ${ }^{3}$ State Key Laboratory of Military Stomatology, National Clinical Research Center for Oral Diseases \& Shaanxi Engineering Research Center for Dental Materials and Advanced Manufacture, Department of Oral Implants, School of Stomatology, The Fourth Military Medical University, Xi'an, People's Republic of China

*These authors contributed equally to this work
\end{abstract}

Correspondence: Hongbo Wei State Key Laboratory of Military Stomatology, National Clinical Research Center for Oral Diseases \& Shaanxi Engineering Research Center for Dental Materials and Advanced Manufacture, Department of Oral Implants, School of Stomatology, The Fourth Military Medical University, No 145 West Changle Road, Xi'an 7I0032, Shaanxi Province, People's Republic of China

Tel +86298477 6454

Fax +86 2984776454

Email weihongbol0I@gmail.com

Guofeng Wu

Department of Prosthodontics, Nanjing Stomatological Hospital, Medical School of Nanjing University, No 30 Zhong Yang Road, Nanjing 210008, Jiangsu, People's Republic of China

Tel +86298477 6456

Fax +862984776456

Email9529612@qq.com

\begin{abstract}
Although percutaneous titanium implants have become one of the best choices as retainers in the facial defects, peri-implantitis still occurs at a significant rate. This unwanted complication occurs due to adhesion of bacteria and subsequent biofilm formation. To solve this problem, we have developed a novel antibiotic nanodelivery system based on self-decomposable silica nanoparticles. In this study, silica-gentamycin (SG) nanoparticles were successfully fabricated using an innovative one-pot solution. The nanoparticles were incorporated within a gelatin matrix and cross-linked on microarc-oxidized titanium. To characterize the SG nanoparticles, their particle size, zeta potential, surface morphology, in vitro drug release, and decomposition process were sequentially evaluated. The antibacterial properties against the gram-positive Staphylococcus aureus, including bacterial viability, antibacterial rate, and bacteria morphology, were analyzed using SG-loaded titanium specimens. Any possible influence of released gentamycin on the viability of human fibroblasts, which are the main component of soft tissues, was investigated. SG nanoparticles from the antibacterial titanium coating continuously released gentamycin and inhibited $S$. aureus growth. In vitro investigation showed that the obtained nanodelivery system has good biocompatibility. Therefore, this design can be further investigated as a method to prevent infection around percutaneous implants.
\end{abstract}

Keywords: silica nanoparticles, microarc oxidation, gentamycin, control release, fibroblasts

\section{Introduction}

The number of patients with acquired or congenital defects in the cranio/maxillofacial region is quite considerable. For these individuals, the use of commercially pure titanium implant-retained facial prosthesis has become the state-of-the-art treatment. ${ }^{1-4}$ Nevertheless, there is no guarantee that these extraoral prostheses will always survive. ${ }^{5}$ Clinical researches have revealed that up to $15 \%-20 \%$ of rehabilitation patients suffered from peri-implantitis due to percutaneous problems. ${ }^{6-8}$ When placed in irradiated sites, the survival rate of the percutaneous implants was only $72.7 \%$. ${ }^{9}$ Previous studies have demonstrated that the major factor determining percutaneous implant success is the formation of a stable skin-implant seal where the implant penetrates the skin. ${ }^{10}$ If the healing process is hampered by the ingress of bacteria into the tissue, it can lead to a localized infection of the implants, soft tissue damage, and ultimately implant failure. ${ }^{11,12}$ This undesirable outcome brings overwhelming troubles for both clinicians and patients. ${ }^{13}$ Therefore, it is imperative that a solution be found to functionalize percutaneous devices with anti-infection abilities.

Effective protocols for the systemic administration of antibiotics for infection prophylaxis have been well established. However, apart from severe toxic side effects, effective local drug levels are difficult to achieve at the target site when drugs are applied systemically. ${ }^{14}$ Controlled/sustained release technology is an effective and 
ideal method to deliver drugs to specific sites, optimize drug dosage, and prolong the delivery duration. Over the past few decades, the incorporation of antimicrobial agents, including antimicrobial peptides, cationic polymers, and inorganic metallic nanoparticles, at the location of the titanium implants has been sparsely investigated. ${ }^{15-20}$ These methods have been well established to possess sustained antimicrobial properties. For instance, Chen et al successfully incorporated titanium implant surfaces with an antimicrobial peptide melamine coating that significantly reduced biofilm formation. ${ }^{21}$ Many studies have focused on developing silver nanoparticles immobilized on titanium surfaces. ${ }^{18,22}$ However, these methods suffer from limitations, such as drug decomposition or an imperfection distribution. A few studies have examined the antimicrobial characteristics of percutaneous devices in the facial area.

Silica nanoparticles are known for their significant biocompatibility as well as ease of surface modification and have a variety of functionalities that can be used to control drug loading and the rate of drug release. ${ }^{23}$ The product of silica degradation is nontoxic silicic acid, which acts as a source of $\mathrm{Si}$ for the formation of connective tissues. ${ }^{24}$ Recently, a new silica-based self-decomposable nanodrug-delivery system has been reported..$^{25,26}$ This drug carrier does not require complicated organic synthesis and dose not result in rapid degradation and premature drug release. ${ }^{27}$ Herein, we report an innovative antibiotic nanodelivery system based on self-decomposable silica nanoparticles for percutaneous implant infection prophylaxis. As a model antibiotic for the present investigation, we chose gentamycin sulfate as the drug to be incorporated during nanoparticle growth. Silica-gentamycin (SG) nanoparticles were incorporated within a gelatin matrix and cross-linked on microarc-oxidized (MAO) titanium. ${ }^{28}$

In this study, we evaluated the characteristics of the nanoparticle system. Gentamycin release from the SG nanoparticles in vitro was determined. The antibacterial properties and possible influence of released gentamycin on the viability of cells were analyzed.

\section{Materials and methods}

\section{Fabrication and characterization of SG nanoparticles}

All chemicals were obtained from Sigma-Aldrich (St Louis, MO, USA) unless otherwise stated and were used without further purification. SG nanoparticles were fabricated according to the modified Stöber method described in a previous study. ${ }^{29}$ Briefly, $20 \mathrm{mg}$ of gentamycin sulfate was dissolved in a mixture of $75 \mathrm{~mL}$ of ethanol (FUYU Fine
Chemicals Co., Ltd., Tianjin, People's Republic of China) with $3.4 \mathrm{~mL}$ of a $25 \%$ ammonia-water solution (FUYU Fine Chemicals Co., Ltd.). To this solution $0.2 \mathrm{~mL}$ of tetraethyl orthosilicate was added slowly with vigorous stirring, and the final mixture was stirred for 24 hours at room temperature. The resulting product was washed with deionized water at least four times and freeze dried.

The shape and morphology of the SG nanoparticles were observed by scanning electron microscopy (SEM, S-4800; Hitachi High Technologies, Tokyo, Japan) and transmission electron microscopy (TEM, Tecnai G2; FEI Company, Hillsboro, OR, USA). A particle size analyzer (DelsaNano C; Beckman Coulter, Brea, CA, USA) was used to assess the mean particle size, polydispersity index, and zeta potential of the nanoparticles at $25^{\circ} \mathrm{C}$ using samples appropriately dispersed in deionized water.

\section{In vitro release test and decomposition process of SG nanoparticles}

To evaluate the drug release behavior of the materials, $20 \mathrm{mg}$ of SG samples was placed into a dialysis bag (molecular weight cutoff $100 \mathrm{kDa}$, Shanghai Green Bird Science and Technology Development Co., Ltd., Shanghai, People's Republic of China), immersed in $50 \mathrm{~mL}$ of phosphatebuffered saline (PBS), and incubated at $37^{\circ} \mathrm{C}$. The $\mathrm{pH}$ of the buffered solution was adjusted to 7.4. At predetermined time points, $1 \mathrm{~mL}$ aliquots of the release medium were taken for gentamycin analysis, with an equal amount of fresh buffer. All release media samples were stored at $4^{\circ} \mathrm{C}$ prior to quantification with an enzyme-linked immunosorbent assay kit.

The degradation of SG was monitored by morphological investigation using TEM.

\section{Preparation of titanium specimens}

\section{Smooth polished titanium}

Disk-shaped commercially pure titanium sheets $(10 \mathrm{~mm}$ in diameter and $2 \mathrm{~mm}$ thick; Northwest Institute for Non-ferrous Metal Research, Xi'an, People's Republic of China) were mechanically ground using various grades of silicon carbide paper (400-7,000 grit) and then ultrasonically cleaned in acetone (FUYU Fine Chemicals Co., Ltd.), ethanol, and deionized water.

\section{MAO substrates}

The MAO procedures took place in an electrolyte containing 0.04 M $\beta$-glycerophosphate sodium (Ruixi Biological Technology Co., Ltd., Xi'an, People's Republic of China) and 0.2 M calcium acetate (Ruixi Biological Technology Co., Ltd.) 
using a pulsed direct current power supply (Northwest Institute for Non-ferrous Metal Research). The applied voltage, frequency, duty cycle, and oxidizing time were $300 \mathrm{~V}, 600 \mathrm{~Hz}, 8.0 \%$, and 5 minutes, respectively. ${ }^{28}$ Immediately after oxidation, the specimens were rinsed with deionized water and air dried.

\section{Cross-linked gelatin/SG composite coating on MAO substrates}

In a typical synthesis of the antibacterial coating on a MAO substrate, $2 \mathrm{mg}$ of $\mathrm{SG}$ nanoparticles were dispersed in $1 \mathrm{~mL}$ of $0.1 \%$ gelatin solution $(\mathrm{w} / \mathrm{v})$, and $0.2 \mathrm{~mL}$ of the suspension was added to the MAO specimen. The substrates were then oscillated on an oscillator for 30 minutes to enable the nanoparticles to be situated in the MAO titanium pores..$^{30}$ This procedure was repeated twice, and the specimen was dried at $4^{\circ} \mathrm{C}$. Via immersing in a $2.5 \%(\mathrm{w} / \mathrm{v})$ glutaraldehyde solution (FUYU Fine Chemicals Co., Ltd.) for 30 minutes, the gelatin in the coating was cross-linked. Finally, the samples were washed three times with ethanol.

The surface structures of the smooth polished (S-Ti), MAO-Ti, and SG nanoparticles cross-linked (SG-Ti) titanium specimens were analyzed using SEM, wherein S-Ti served as the control.

\section{Antibacterial effect of gelatin/SG composite coating on MAO substrates} Antibacterial rate

Staphylococcus aureus (ATCC 25923; ATCC, Manassas, VA, USA) was grown to mid-logarithmic phase in MuellerHinton medium (Aobox Biotechnology, Beijing, People's Republic of China). The bacterial suspension was diluted to a concentration of $10^{6} \mathrm{CFU} / \mathrm{mL}$. The specimens (S-Ti, MAO-Ti, and SG-Ti) were separately incubated in $1 \mathrm{~mL}$ of the bacterial suspension at $37^{\circ} \mathrm{C}$ for 24 hours. Following incubation, the various titanium samples were rinsed twice with PBS. The bacteria attached to the specimens were detached using $5 \mathrm{~mL}$ of PBS for 5 minutes. The bacterial suspensions were cultivated on Mueller-Hinton agar plates for colony counting. The antibacterial rate (AR) was calculated according to the following formula: $\mathrm{AR}(\%)=\left(\mathrm{CFU}_{\text {control }}\right.$ $\mathrm{CFU}_{\text {experiment }} / \mathrm{CFU}_{\text {control }} \times 100 \%$, where S-Ti is the control, and $\mathrm{S}-\mathrm{Ti}$ and SG-Ti are the experimental groups.

\section{Bacterial viability}

The samples (S-Ti, MAO-Ti, and SG-Ti) were incubated with $1 \mathrm{~mL}$ of the bacteria suspension $\left(10^{6} \mathrm{CFU} / \mathrm{mL}\right)$ for 24 hours and then rinsed three times with PBS. The specimens were stained with SYTO 9 and propidium iodide dyes (LIVE/ DEAD BacLight ${ }^{\mathrm{TM}}$ Bacterial Viability Kits L13152; Life Technologies Corp, Carlsbad, CA, USA) for 15 minutes in the dark. Live bacteria were stained by SYTO 9 (green), and the dead bacteria (with damaged membranes) were stained by propidium iodide (red) for examination using laser scanning confocal microscopy (FV1000; Olympus, Tokyo, Japan).

\section{Bacterial morphology}

After incubation with $1 \mathrm{~mL}$ of the bacteria suspension $\left(10^{6} \mathrm{CFU} / \mathrm{mL}\right)$ for 24 hours, the titanium samples ( $\mathrm{S}-\mathrm{Ti}$, MAO-Ti, and SG-Ti) were rinsed twice with PBS, fixed with $3 \%$ glutaraldehyde at $4{ }^{\circ} \mathrm{C}$ overnight, and dehydrated in a series of ethanol solutions for 10 minutes each. The specimens were dried, platinum coated, and examined by SEM to observe the bacterial morphology.

\section{Study of the interaction of titanium samples with human cells \\ Cell culture}

Primary human skin fibroblasts were isolated as previously described. ${ }^{31}$ The cells were cultured in low glucose Dulbecco's Modified Eagle's Medium (DMEM; Thermo Fisher Scientific Inc., Pittsburg, PA, USA) containing 10\% fetal bovine serum (Thermo Fisher Scientific Inc.) and 1\% penicillin/streptomycin (Thermo Fisher Scientific Inc.) in a humidified atmosphere of $5 \% \mathrm{CO}_{2}$ at $37^{\circ} \mathrm{C}$.

\section{Cell morphology}

The cells were seeded onto the experimental substrates (S-Ti, MAO-Ti, and SG-Ti) when the density reached a suitable population. After 1 day of culture, the specimens were rinsed twice with PBS, fixed with $3 \%$ glutaraldehyde at $4{ }^{\circ} \mathrm{C}$ overnight, and dehydrated in a series of ethanol solutions. The specimens were dried, platinum coated, and examined by SEM to observe the morphological spread of the fibroblasts.

\section{Cytotoxicity of materials}

The cell counting kit-8 (CCK-8) (Dojindo Laboratories, Kumamoto, Japan) method was performed according to the manufacturer's instructions. Briefly, $200 \mu \mathrm{L}$ of fibroblast suspensions $(5,000$ cells $/ \mathrm{mL})$ was seeded into each well of a 96-well cell culture plate. After 24 hours of incubation at $37^{\circ} \mathrm{C}$ under $5 \% \mathrm{CO}_{2}$ for cell attachment, the medium was replaced by different material extracts at different concentrations (prepared by soaking each experimental substrate in $1 \mathrm{~mL}$ of cell culture medium at $37^{\circ} \mathrm{C}$ for 24 hours, then 
diluting with 0,1 , or $4 \mathrm{~mL}$ of DMEM). Each of the described groups required six wells. After 3 days of incubation, the media in each well were removed and $100 \mu \mathrm{L}$ of fresh culture medium was added to each well. To each well $10 \mu \mathrm{L}$ of CCK-8 solution was added, and the plate was incubated for 2 hours at $37^{\circ} \mathrm{C}$. The absorbance was measured at $450 \mathrm{~nm}$ using a microplate reader $\left(\right.$ Synergy $^{\text {TM }}$ HT, BioTek Instruments, Inc., Winooski, VT, USA). The wells containing fibroblasts without extracts were used as controls.

\section{Statistical analysis}

All experiments were repeated for a minimum of three times, and data are expressed as the mean \pm standard deviation. To determine the level of significance, a one-way analysis of variance combined with Student's $t$-test was used; statistical significance was considered at $P<0.05$.

\section{Results}

\section{Characterization of SG nanoparticles}

SEM (Figure 1A) and TEM (Figure 1B) images show that SG nanoparticles were spherical in shape, with a diameter of $\sim 250 \mathrm{~nm}$. As already mentioned, the particle size was also determined with a particle size analyzer. It is estimated that the logarithmic particle size distribution was normal and the average particle size was $298.1 \pm 12.2 \mathrm{~nm}$. The polydispersity index and zeta potential were $0.187 \pm 0.012$ and $-42.64 \pm 1.10 \mathrm{mV}$, respectively.

\section{In vitro release test and decomposition process of SG nanoparticles}

As shown in Figure 2, a prolonged release pattern was observed for gentamycin, following a faster release in the first 12 hours in which $29.40 \%$ of the drug was released. Overall, $88.22 \%$ of the accumulated drug release was achieved after 14 days. The biphasic release provides a high concentration of gentamycin at first, and then, a slow release of drug maintains the concentration of gentamycin at the therapeutic level.

The decomposition process of the SG nanoparticles was observed using TEM (Figure 3A-D). A hollow feature appeared in the center of some nanoparticles after 3 days of immersion. The number of hollow-centered nanoparticles increased in the subsequent days, and some particles collapsed due to thinner shells.

\section{Characterization of titanium specimens}

Representative SEM images of the different titanium specimens are shown in Figure 4. The unmodified titanium surface was relatively smooth after being polished with silicon carbide paper (Figure 4A). A porous morphology with pores of $1-5 \mu \mathrm{m}$ in diameter appeared after MAO treatment (Figure 4B). These evenly distributed pores provided a space for the attachment and loading of SG nanoparticles. As seen in Figure 4C and D, SG nanoparticle was filled in the pores on MAO-Ti after oscillation and cross-linking.

\section{Antibacterial effects}

Antibacterial effects were first assessed by a quantitative method. The bacteria colony count on the surface of SG-Ti specimens was significantly low compared to that of MAO-Ti and S-Ti specimens (Figure 5). The quantification of the number of colonies demonstrated that the SG-Ti specimens possessed a positive and high AR value of $\sim 86.35 \%$. However, the MAO-Ti specimen had an AR value of $\sim-270.32 \%$.

The fluorescence staining results were consistent with the quantitative results. As shown in Figure 6, after 24 hours of incubation, the number of viable bacteria (green fluorescence) adhered to SG-Ti (Figure 6C) was far less than that of $\mathrm{S}-\mathrm{Ti}$ (Figure 6A) and MAO-Ti (Figure 6B). In contrast, large
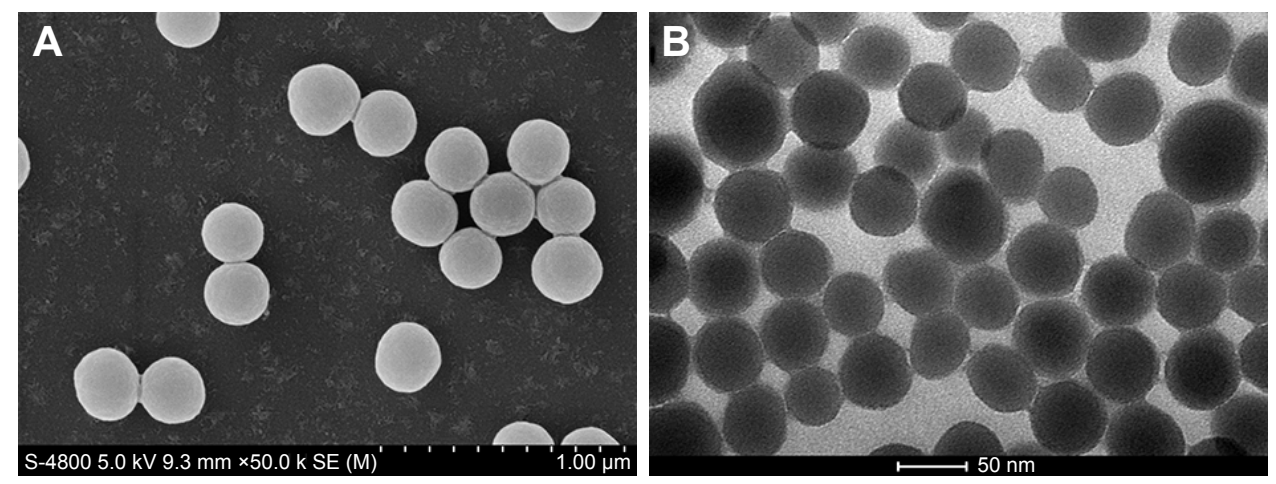

Figure I (A) SEM images of SG nanoparticles, (B) TEM images of SG nanoparticles.

Abbreviations: SEM, scanning electron microscopy; SG, silica-gentamycin; TEM, transmission electron microscopy. 


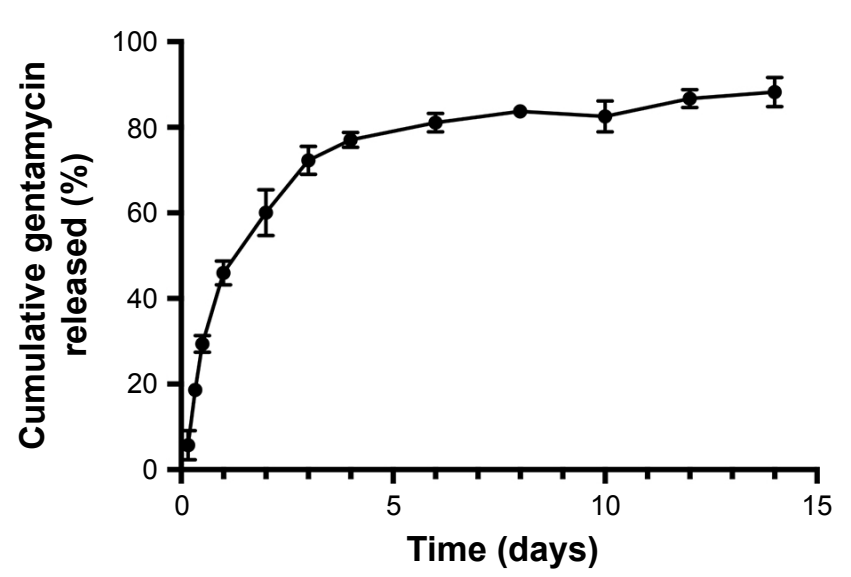

Figure 2 Release profile for SG nanoparticles soaked in PBS over 14 days. Abbreviations: SG, silica-gentamycin; PBS, phosphate-buffered saline.

amounts of dead bacteria (red fluorescence) were observed on SG-Ti (Figure 6C).

SEM images showed that a large number of $S$. aureus colonies persisted on the S-Ti and MAO-Ti specimens (Figure 7), and lower levels of bacteria colonization were observed on SG-Ti (Figure 7C). Some of the bacteria in direct contact with the SG nanoparticles exhibited perforated or deformed membranes.

\section{Cytotoxicity of materials}

All tested specimens presented good biocompatibility properties, as revealed by microscopic analysis and the CCK- 8 test. Figure 8 shows the morphology of human fibroblasts spread on the three surfaces. Generally, the fibroblasts were firmly attached and well spread on all specimens. They showed a fusiform morphology, with some filopodia and lamellipodia extensions.

The CCK-8 test revealed that there was no significant difference in cytotoxicity among different extract concentrations for each of the three groups (Figure 9).

\section{Discussion}

Peri-implantitis around titanium percutaneous implants mainly occurs due to bacterial adhesion and biofilm accumulation. Conventional smooth percutaneous titanium devices become surrounded with a thick fibrotic capsule after
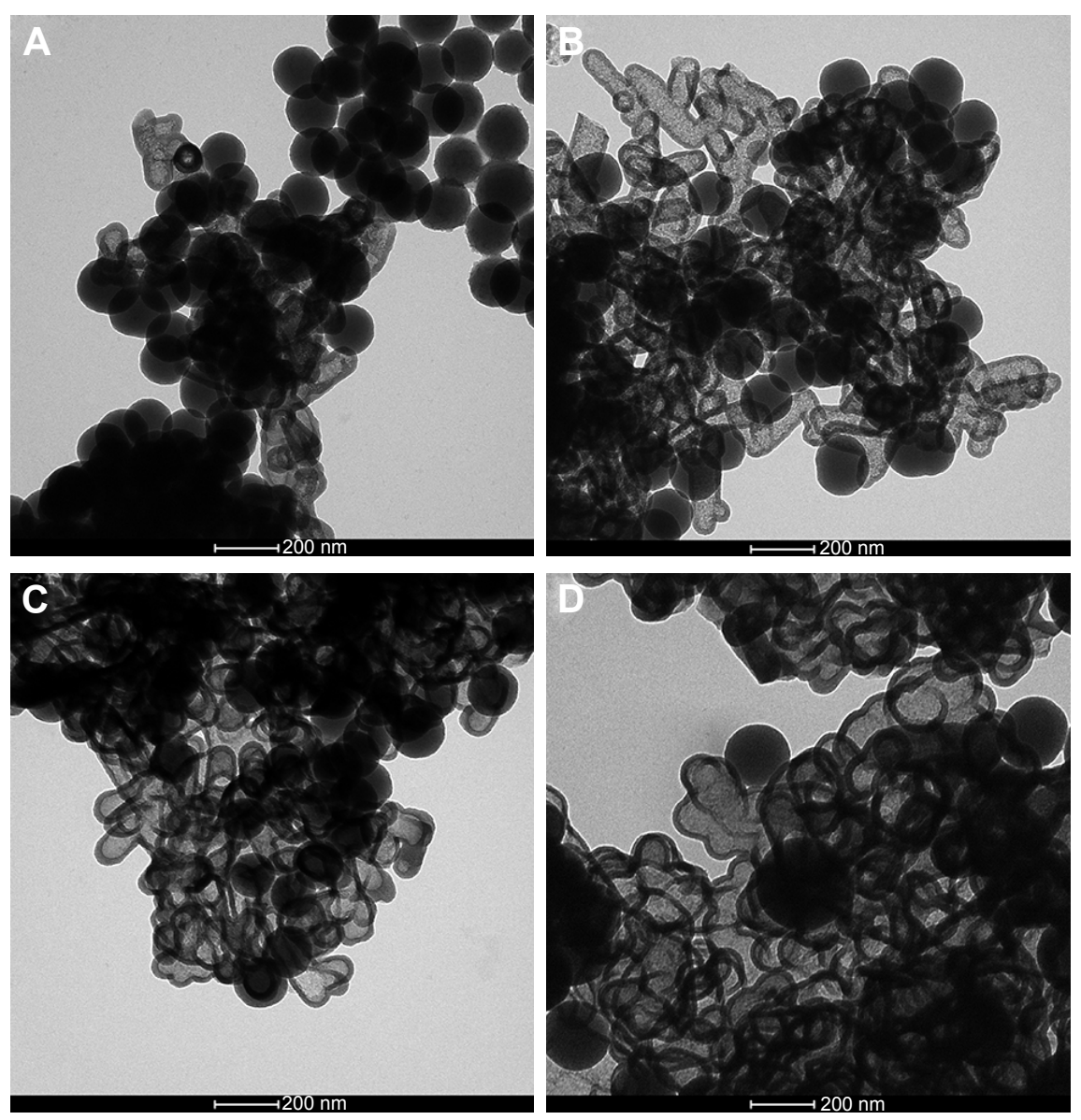

Figure 3 TEM images of SG nanoparticles immersed in PBS after (A) 3, (B) 7, (C) 10, and (D) 14 days.

Abbreviations: TEM, transmission electron microscopy; SG, silica-gentamycin; PBS, phosphate-buffered saline. 

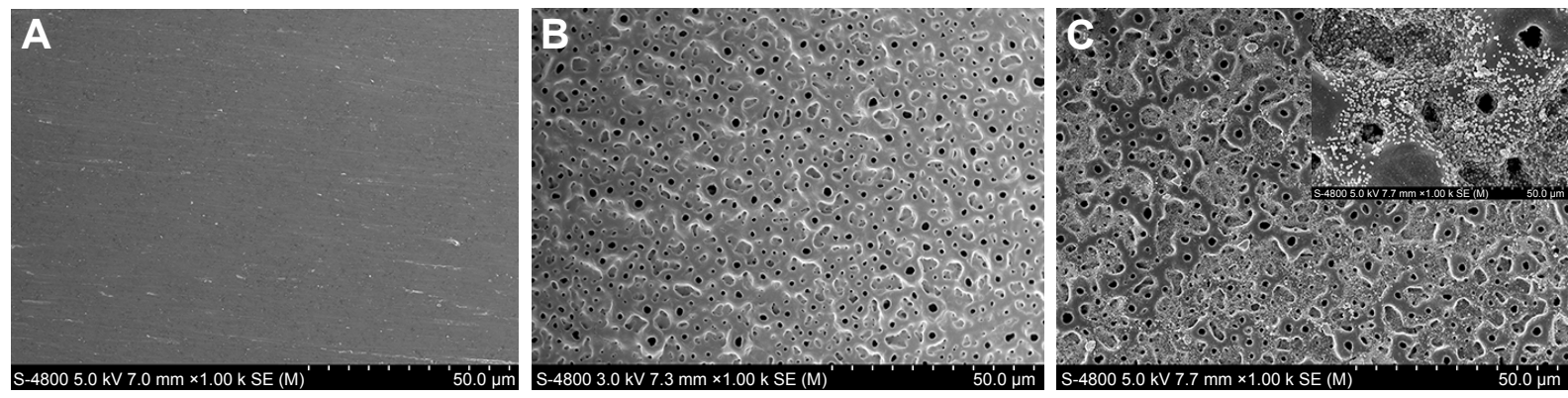

Figure 4 SEM images of (A) smooth titanium surface, (B) titanium surface after microarc-oxidized treatment, (C) titanium surface with antibacterial coating under low magnification, and (inset) under high magnification.

Abbreviation: SEM, scanning electron microscopy.

implantation; these structures are inadequate for providing a long-term seal to defend against bacterial invasion..$^{32}$ Therefore, to form a favorable percutaneous seal and to increase the stability of implants, titanium surfaces are imbued with anti-bacterial properties. Surface coating techniques, which modify the surface components and structure of materials, are among the best options for titanium surface enhancement. Many drugs and coatings have been developed to create antibacterial surfaces that either kill bacteria or prevent their attachment to implant surfaces. ${ }^{33}$ However, there is a need to develop percutaneous devices with good soft tissue biocompatibility that can further reduce peri-implantitis in a cost-effective manner.

Among inorganic-based nanomaterials, silica nanoparticles have several attractive features such as controllable particle sizes and shapes, ease of large-scale synthesis, and excellent biocompatibility and stability. ${ }^{23}$ Many molecules have been successfully loaded onto silica nanoparticles, which serve as sustained drug delivery systems. The most common procedure to load drugs involves soaking the nanoparticles in a highly concentrated drug solution, followed by drying, which is both time-consuming and complex; ${ }^{34}$ a one-pot synthetic route would be preferable. Recently, a novel type of self-decomposable silica nanoparticle was reported..$^{29}$ Drug molecules were encapsulated in the nanoparticle during preparation under mild conditions. ${ }^{29}$ To our knowledge, no other reports exists on the application of selfdecomposing silica nanoparticles as an innovative antibiotic nanodelivery system in the field of biomedical implants.

In this study, we successfully incorporated gentamycin into these novel silica nanoparticles. SEM and TEM investigations showed that the SG nanoparticles were spherical in shape, with a diameter of $\sim 250 \mathrm{~nm}$. Nanospheres of this size easily fit into the volcanic-like holes of MAO surfaces. According to Vasilev et al the ideal release profile should provide a fast initial release within the first 6 hours, which protects the site while the immune system is weakened, followed by a slow release. ${ }^{35}$ Our release profile results and TEM investigation of the SG nanoparticles during 2 weeks were consistent with this time course. When the silica was decomposed, a fast release over the first 8 hours provided a high concentration of gentamycin and the subsequent slow release of the drug maintained the concentration of gentamycin at a therapeutic level. This biphasic profile can play an important role against infection in the early implant stage. When the gelatin on the titanium coating degraded progressively, the nanoparticles were subsequently released. ${ }^{30}$ As a consequence, when the SG nanoparticles were fixed in the
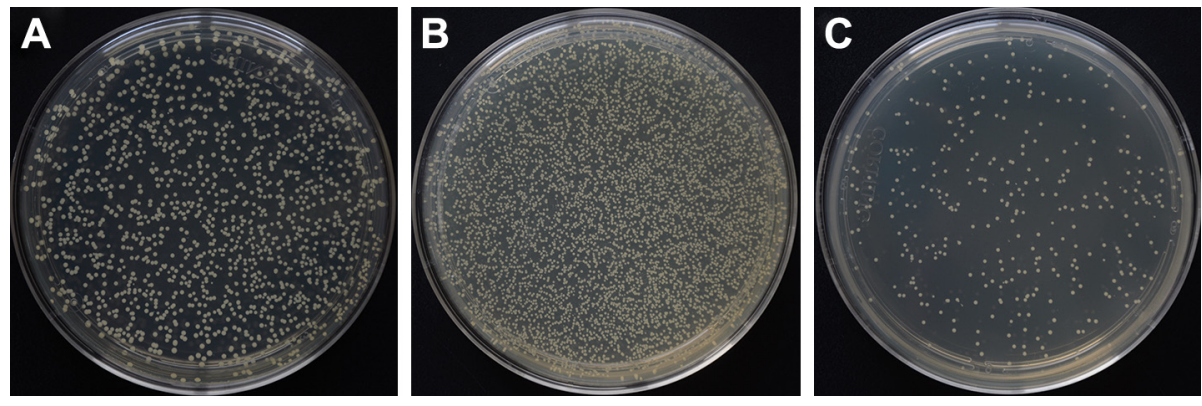

Figure 5 Bacteria colonies cultivated on Mueller-Hinton agar plates from (A) S-Ti, (B) MAO-Ti, and (C) SG-Ti groups.

Abbreviations: S-Ti, smooth polished titanium; MAO-Ti, microarc-oxidized titanium; SG-Ti, silica nanoparticles cross-linked titanium. 

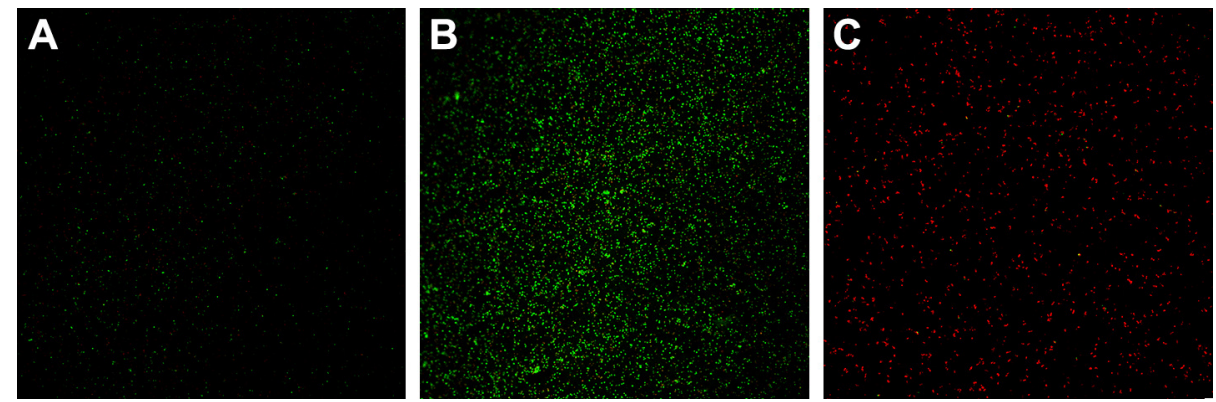

Figure 6 Staphylococcus aureus adherence on the (A) S-Ti, (B) MAO-Ti, and (C) SG-Ti specimens as observed by fluorescence microscopy. Abbreviations: S-Ti, smooth polished titanium; MAO-Ti, microarc-oxidized titanium; SG-Ti, silica nanoparticles cross-linked titanium.

titanium MAO coating by a gelatin matrix, the release time was prolonged. Our design not only provided an innovative antibiotic coating for the treatment of bacterial infection with a minimum dose of antibiotics, but also prolonged the duration of action as well.

Considering that biofilm formation of bacteria after adhesion to the implant surface is critical for infection, ${ }^{36,37}$ the adhesion of bacteria to different titanium specimens was investigated. $S$. aureus, a gram-positive bacterium that is most commonly isolated with biofilm-associated implant infections, was selected for this experiment. Incubation of the titanium specimens in a culture of $S$. aureus for 24 hours was sufficient to initiate biofilm formation. The method used to assess the anti-biofilm activity on the obtained structures involved quantification of the viable CFU counting, bacterial viability determined by fluorescence staining, and SEM investigation. By killing the bacteria after they contact the SG nanoparticles, SG-Ti exhibited an antimicrobial activity superior to that of MAO-Ti and S-Ti alone. The antibacterial efficacy was not affected by the gelatin process.

Cytocompatibility is a fundamental requirement for further clinical use, and no cytotoxic activity of SG-Ti against
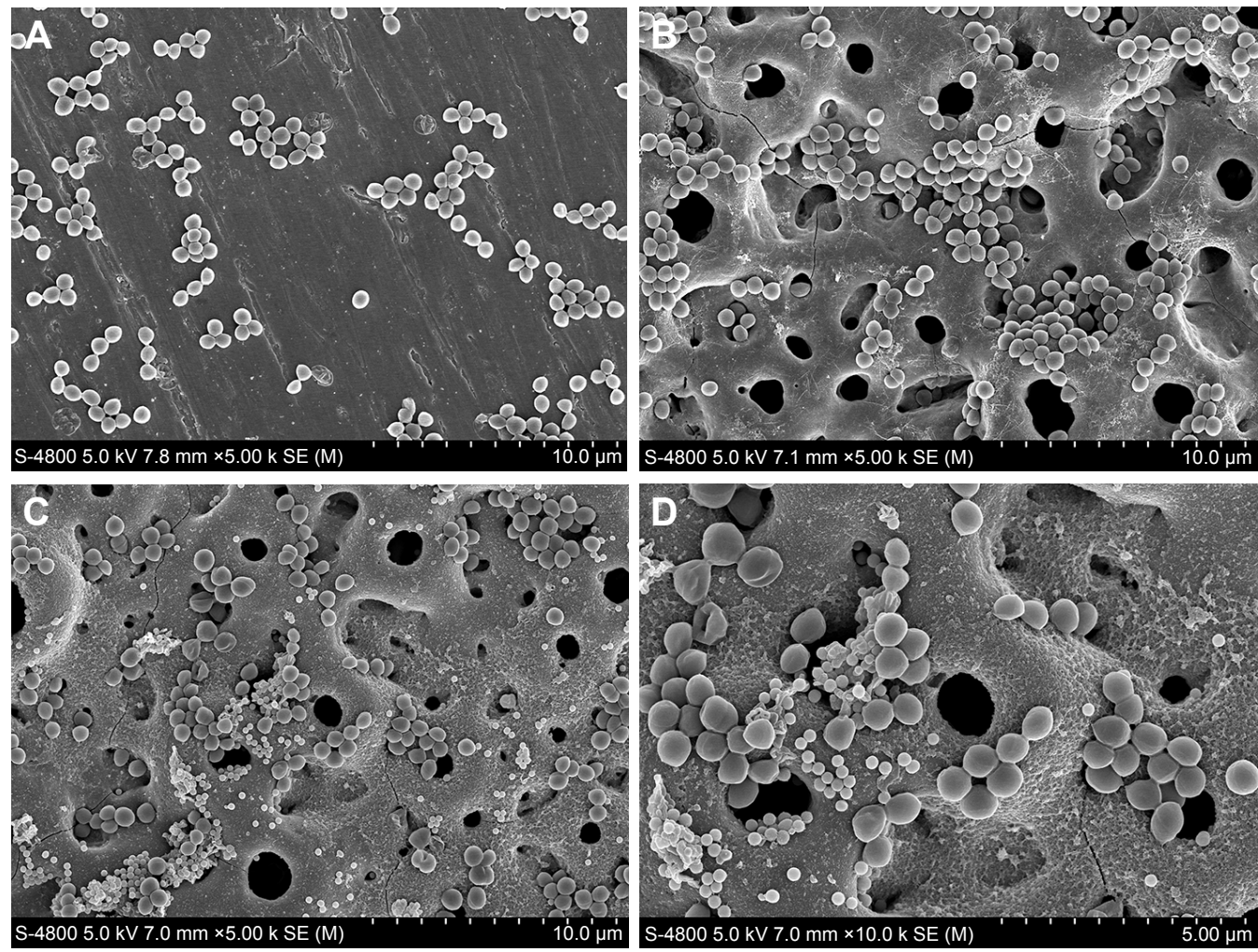

Figure 7 SEM images of Staphylococcus aureus colonized on (A) S-Ti, (B) MAO-Ti, and (C) SG-Ti under low magnification and (D) bacteria with deformed membranes under higher magnification.

Abbreviations: SEM, scanning electron microscopy; S-Ti, smooth polished titanium; MAO-Ti, microarc-oxidized titanium; SG-Ti, silica nanoparticles cross-linked titanium. 

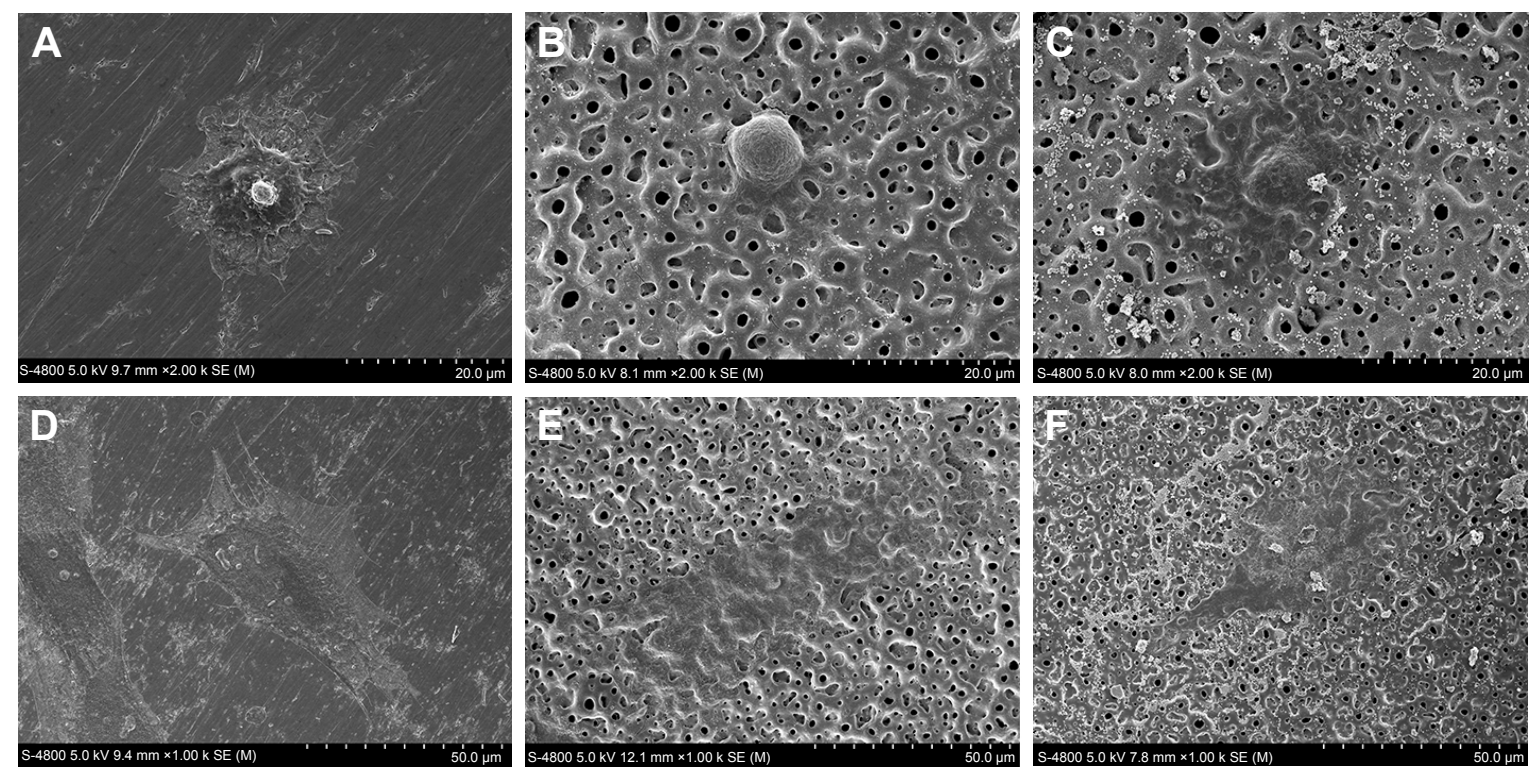

Figure 8 Representative SEM imaged of human fibroblasts spread on (A) S-Ti after 2 hours, (B) MAO-Ti after 2 hours, (C) SG-Ti after 2 hours, (D) S-Ti after 24 hours, (E) MAO-Ti after 24 hours, and (F) SG-Ti after 24 hours.

Abbreviations: SEM, scanning electron microscopy; S-Ti, smooth polished titanium; MAO-Ti, microarc-oxidized titanium; SG-Ti, silica nanoparticles cross-linked titanium.

human cells was observed in our investigation. The SG nanoparticles were rather safe for human tissues, and the delayed drug release from the particles had no effect on nearby tissue.

In summary, our results suggest a viable solution for novel percutaneous implants with a significantly lower risk of infection compared to smooth devices. The antimicrobial activity and biocompatibility of the antibacterial titanium coating in vivo will be reported in an upcoming publication.

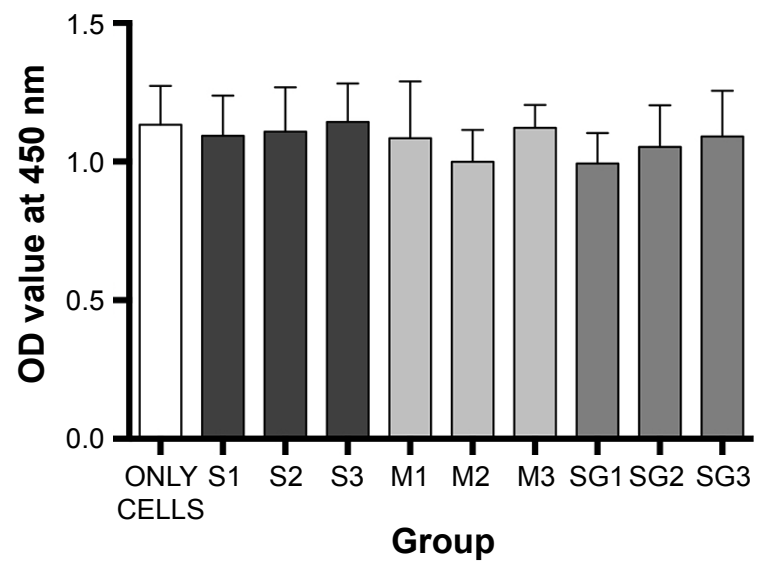

Figure 9 Cell viability on S-Ti, MAO-Ti, and SG-Ti.

Notes: ONLY CELLS represent wells with cells but no added titanium extracts; $\mathrm{SI}-3$ : wells with $\mathrm{S}-\mathrm{Ti}$ in I $\mathrm{mL}$ cell culture medium at $37^{\circ} \mathrm{C}$ for 24 hours, then diluted with 0 , I, or $4 \mathrm{~mL}$ DMEM; MI-3: wells with MAO-Ti in I mL cell culture medium at $37^{\circ} \mathrm{C}$ for 24 hours, then diluted with 0 , I, or $4 \mathrm{~mL}$ DMEM; SGI-3: wells with SG-Ti in I mL cell culture medium at $37^{\circ} \mathrm{C}$ for 24 hours, then diluted with 0 , I, or $4 \mathrm{~mL}$ DMEM.

Abbreviations: S-Ti, smooth polished titanium; MAO-Ti, microarc-oxidized titanium; SG-Ti, silica nanoparticles cross-linked titanium; DMEM, Dulbecco's Modified Eagle's Medium.
Since most biofilms consist of multiple species, the antibacterial ability of the specimens to other species, including gram-negative bacteria, will be further investigated.

\section{Conclusion}

In the present study, we succeeded in developing an innovative local drug release system based on self-decomposable silica nanoparticles. After incorporating SG nanoparticles in the pores of MAO titanium using cross-linked gelatin, we achieved an effective antibacterial coating on the titanium substrate. This antimicrobial coating enabled stable storage and tunable release of gentamycin, displaying potency against gram-positive S. aureus at the same time. Moreover, the released gentamycin was biocompatible with human fibroblasts. The result suggested that this novel antibacterial coating might provide a beneficial alternative when designing percutaneous implants.

\section{Acknowledgment}

Financial support was provided by the National Natural Science Foundation of China (No. 81300918).

\section{Disclosure}

The authors report no conflicts of interest in this work.

\section{References}

1. Gitto CA, Plata WG, Schaaf NG. Evaluation of the peri-implant epithelial tissue of percutaneous implant abutments supporting maxillofacial prostheses. Int J Oral Maxillofac Implants. 1994;9(2):197-206. 
2. Pekkan G, Tuna SH, Oghan F. Extraoral prostheses using extraoral implants. Int J Oral Maxillofac Surg. 2011;40(4):378-383.

3. Leonardi A, Buonaccorsi S, Pellacchia V, Moricca LM, Indrizzi E, Fini G. Maxillofacial prosthetic rehabilitation using extraoral implants. J Craniofac Surg. 2008;19(2):398-405.

4. Visser A, Raghoebar GM, van Oort RP, Vissink A. Fate of implantretained craniofacial prostheses: life span and aftercare. Int J Oral Maxillofac Implants. 2008;23(1):89-98.

5. Karakoca S, Aydin C, Yilmaz H, Bal BT. Retrospective study of treatment outcomes with implant-retained extraoral prostheses: survival rates and prosthetic complications. J Prosthet Dent. 2010;103(2):118-126.

6. Roumanas ED, Freymiller EG, Chang TL, Aghaloo T, Beumer J 3rd. Implant-retained prostheses for facial defects: an up to 14-year follow-up report on the survival rates of implants at UCLA. Int J Prosthodont 2002;15(4):325-332.

7. Curi MM, Oliveira MF, Molina G, et al. Extraoral implants in the rehabilitation of craniofacial defects: implant and prosthesis survival rates and peri-implant soft tissue evaluation. J Oral Maxillofac Surg. 2012; 70(7):1551-1557.

8. Miles BA, Sinn DP, Gion GG. Experience with cranial implant-based prosthetic reconstruction. J Craniofac Surg. 2006;17(5):889-897.

9. Karakoca S, Aydin C, Yilmaz H, Bal BT. Survival rates and periimplant soft tissue evaluation of extraoral implants over a mean follow-up period of three years. J Prosthet Dent. 2008;100(6):458-464.

10. Jansen JA, van der Waerden JP, de Groot K. Epithelial reaction to percutaneous implant materials: in vitro and in vivo experiments. J Invest Surg. 1989;2(1):29-49.

11. Jansen JA, van der Waerden JP, de Groot K. Fibroblast and epithelial cell interactions with surface-treated implant materials. Biomaterials. 1991;12(1):25-31.

12. Holt BM, Betz DH, Ford TA, Beck JP, Bloebaum RD, Jeyapalina S. Pig dorsum model for examining impaired wound healing at the skinimplant interface of percutaneous devices. J Mater Sci Mater Med.2013; 24(9):2181-2193.

13. Darouiche RO. Treatment of infections associated with surgical implants. N Engl J Med. 2004;350(14):1422-1429.

14. Vester H, Wildemann B, Schmidmaier G, Stöckle U, Lucke M. Gentamycin delivered from a PDLLA coating of metallic implants: in vivo and in vitro characterisation for local prophylaxis of implantrelated osteomyelitis. Injury. 2010;41(10):1053-1059.

15. de Breij A, Riool M, Kwakman PH, et al. Prevention of Staphylococcus aureus biomaterial-associated infections using a polymer-lipid coating containing the antimicrobial peptide OP-145. J Control Release. 2016; 222:1-8

16. Choe H, Narayanan AS, Gandhi DA, et al. Immunomodulatory peptide IDR-1018 decreases implant infection and preserves osseointegration. Clin Orthop Relat Res. 2015;473(9):2898-2907.

17. Calliess T, Sluszniak M, Winkel A, et al. Antimicrobial surface coatings for a permanent percutaneous passage in the concept of osseointegrated extremity prosthesis. Biomed Tech (Berl). 2012;57(6):467-471.

18. Mei S, Wang H, Wang W, et al. Antibacterial effects and biocompatibility of titanium surfaces with graded silver incorporation in titania nanotubes. Biomaterials. 2014;35(14):4255-4265.

19. Abdulkareem EH, Memarzadeh K, Allaker RP, Huang J, Pratten J, Spratt D. Anti-biofilm activity of zinc oxide and hydroxyapatite nanoparticles as dental implant coating materials. J Dent. 2015;43(12):1462-1469.
20. Elizabeth E, Baranwal G, Krishnan AG, Menon D, Nair M. ZnO nanoparticle incorporated nanostructured metallic titanium for increased mesenchymal stem cell response and antibacterial activity. Nanotechnology. 2014;25(11):337-345.

21. Chen R, Willcox MD, Ho KK, Smyth D, Kumar N. Antimicrobial peptide melimine coating for titanium and its in vivo antibacterial activity in rodent subcutaneous infection models. Biomaterials. 2016;85:142-151.

22. Necula BS, van Leeuwen JP, Fratila-Apachitei LE, Zaat SA, Apachitei I, Duszczyk J. In vitro cytotoxicity evaluation of porous $\mathrm{TiO}_{2}-\mathrm{Ag}$ antibacterial coatings for human fetal osteoblasts. Acta Biomater. 2012;8(11): 4191-4197.

23. Yildirim A, Ozgur E, Bayindir M. Impact of mesoporous silica nanoparticle surface functionality on hemolytic activity, thrombogenicity and nonspecific protein adsorption. J Mater Chem B. 2013;1(14):1909-1920.

24. Sripanyakorn S, Jugdaohsingh R, Dissayabutr W, Anderson SH, Thompson RP, Powell JJ. The comparative absorption of silicon from different foods and food supplements. Brit J Nutr. 2009;102(6):825-834.

25. Fan L, Jin B, Zhang S, Song C, Li Q. Stimuli-free programmable drug release for combination chemo-therapy. Nanoscale. 2016;8(25): 12553-12559.

26. Fan L, Zhang Y, Wang F, et al. Multifunctional all-in-one drug delivery systems for tumor targeting and sequential release of three different anti-tumor drugs. Biomaterials. 2016;76:399-407.

27. Zhao S, Zhang S, Ma J, etal. Double loaded self-decomposable $\mathrm{SiO}_{2}$ nanoparticles for sustained drug release. Nanoscale. 2015;7(39):16389-16398.

28. Wei D, Zhou Y, Jia D, Wang Y. Characteristic and in vitro bioactivity of a microarc-oxidized $\mathrm{TiO}(2)$-based coating after chemical treatment. Acta Biomater. 2007;3(5):817-827.

29. Zhang S, Chu Z, Yin C, Zhang C, Lin G, Li Q. Controllable drug release and simultaneously carrier decomposition of SiO2-drug composite nanoparticles. J Am Chem Soc. 2013;135(15):5709-5716.

30. Cheng Y, Wu J, Gao B, et al. Fabrication and in vitro release behavior of a novel antibacterial coating containing halogenated furanone-loaded poly(L-lactic acid) nanoparticles on microarc-oxidized titanium. Int $J$ Nanomed. 2011;7(6):5641-5652.

31. El-Ghalbzouri A, Van Den Bogaerdt AJ, Kempenaar J, Ponec M. Human adipose tissue-derived cells delay re-epithelialization in comparison with skin fibroblasts in organotypic skin culture. Br J Dermatol. 2004; 150(3):444-454.

32. Isackson D, McGill LD, Bachus KN. Percutaneous implants with porous titanium dermal barriers: an in vivo evaluation of infection risk. Med Eng Phys. 2011;33(33):418-426.

33. Actis L, Gaviria L, Guda T, Ong JL. Antimicrobial surfaces for craniofacial implants: state of the art. J Korean Assoc Oral Maxillofac Surg. 2013;39(39):43-54.

34. Izquierdo-Barba I, Vallet-Regí M, Kupferschmidt N, Terasaki O, Schmidtchen A, Malmsten M. Incorporation of antimicrobial compounds in mesoporous silica film monolith. Biomaterials. 2009;30(29): 5729-5736.

35. Vasilev K, Cook J, Griesser HJ. Antibacterial surfaces for biomedical devices. Expert Rev Med Devices. 2009;6(5):553-567.

36. Swartjes JJ, Sharma PK, van Kooten TG, et al. Current developments in antimicrobial surface coatings for biomedical applications. Curr Med Chem. 2015;22(18):2116-2129.

37. Costerton JW, Stewart PS, Greenberg EP. Bacterial biofilms: a common cause of persistent infections. Science. 1999;284(5418):1318-1322.
International Journal of Nanomedicine

\section{Publish your work in this journal}

The International Journal of Nanomedicine is an international, peerreviewed journal focusing on the application of nanotechnology in diagnostics, therapeutics, and drug delivery systems throughou the biomedical field. This journal is indexed on PubMed Central, MedLine, CAS, SciSearch ${ }^{\circledR}$, Current Contents ${ }^{\circledR} /$ Clinical Medicine,

\section{Dovepress}

Journal Citation Reports/Science Edition, EMBase, Scopus and the Elsevier Bibliographic databases. The manuscript management system is completely online and includes a very quick and fair peer-review system, which is all easy to use. Visit http://www.dovepress.com/ testimonials.php to read real quotes from published authors. 